\title{
A Relationship Between Time Perception and State-Anxiety
}

James Brown

Department of Psychology, Coastal Carolina University

jmbrown@g.coastal.edu

\begin{abstract}
Participants in this study were randomly assigned to one of three interval groups of either one, three, or five minutes. All participants were asked to estimate a group-assigned time interval and complete the state portion of the State Trait Anxiety Inventory (STAI). It was hypothesized that higher levels of state anxiety would cause participants to overestimate the passage of time. It was also hypothesized that shorter interval durations would be estimated more accurately than longer interval durations. Results of a $t$ test did not support the first hypothesis. Results of a linear trend analysis did support the second hypothesis $(\mathrm{P}<.05)$. These results indicate that state-anxiety does not cause the passage of time to be overestimated and that interval duration length affects how accurately time is estimated. Implications of this study are important to the understanding of human time-management ability and time estimation errors.
\end{abstract}

\section{KEYWORDS}

Time estimation; Perception; State-anxiety; Attention; Working memory; Emotion

\section{INTRODUCTION}

Our ability to comprehend causal relationships in time is a fundamental component of learning and human behavior. ${ }^{1}$ Humans monitor time by referring to interval durations of seconds, minutes, and hours. Because time is constant, a second will always be a second, an hour an hour, and so forth. However, perception of those interval durations tends to be variable and often inaccurate. ${ }^{2}$ If not for the invention of instruments to measure time, the ability to accurately quantify and monitor how much time has elapsed in the form of some unit of measurement would be quite inconsistent. Unlike most of the neural input the brain receives from the receptors for taste, touch, smell, sight, and hearing, time is not physically sensed, yet the passage of time can be sensed cognitively. $^{3,4}$ According to pacemaker-accumulator models of time perception, time perception is a product of cognitive reinforcement and working memory, and time estimation is made possible by a series of neural signals produced by an internal pacemaker in the brain., ${ }^{3}$ Under pacemaker-accumulator models of time perception, neural signals are collated, counted and compared to stored representations of interval durations to which future durations and time estimations are based (see Figure 1. for depiction of internal time perception model). ${ }^{5}$

\section{MODELS OF TIME PERCEPTION}

Numerous studies continually support cognitive pacemaker-accumulator models of time perception, showing that estimations of durations approximately two seconds or longer rely primarily on working memory and that participants tend to make more time error estimations when they are presented with visual and auditory distractions. ${ }^{6}$ Scalar Expectancy Theory (SET), which is a widely accepted pacemaker-accumulator model of time perception, hypothesizes that intervals of time are perceived when an individual consciously attends to them. ${ }^{3}$ Further, because attention and working memory work conjunctively, any exogenous factors that alter attention, emotional arousal in particular, will also alter the perception of time. ${ }^{7}$ Thus, emotional variables that affect working memory should also affect the perception of time. Decades of electrophysiological studies support this notion, showing that emotional arousal, attention and working memory are neuro-functionally coupled within the anterior cingulate cortex (ACC), a brain structure found to be involved decision-making tasks, impulse control and emotion processing in general. ${ }^{8}$

\section{NEUROPHYSIOLOGICAL CORRELATES}

Providing a proof of concept for a functional link between emotional arousal, attention and working memory is a study that examined the influence of emotional context on attentional resources was indexed using electroencephalogram (EEG). ${ }^{9}$ Eventrelated potentials (ERPs) related to attentional processing and working memory were recorded while participants engaged in a computerized version of the Go/Nogo task modified to include background images either positive, negative or neutral in emotional valence. Statistical analyses of ERPs indicated that the magnitude of ACC signals changed differentially depending on whether participants were exposed to positive or negative emotional stimuli. Further, the presentation of negative emotional stimuli positively correlated with participants having increased reaction time. ${ }^{9}$ 


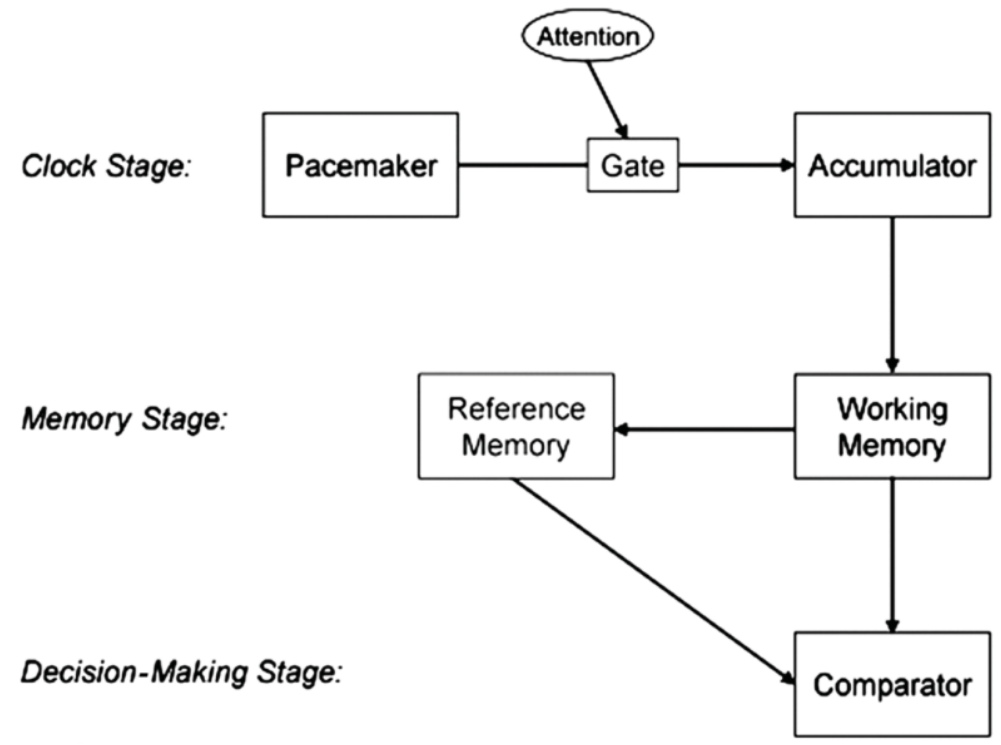

Figure 1. A graphical representation of an internal clock model of time perception. This depiction is from a study by Pencil, Coslett, Aguirre and Chatterjee (2010) and was created to discuss the neural bases for interval timing. ${ }^{5}$

The authors concluded that the neurophysiological effects of high state-anxiety disrupted the attentional resources of the participants because the participants' attentional resources were directed to negative thought processes, thus leading to their performance changes. ${ }^{9}$ Although this was not a time perception study, because time perception is hypothesized to be a process utilizing attentional resources it is reasonable to suggest that if emotional arousal altered attentional processes time perception processes would also have been altered. Previous studies even suggest that meditation, an activity empirically supported to increased awareness and mental clarity, works by engaging the ACC, as well the insula, posterior cingulate cortex (PCC), prefrontal and thalamic cortices, such that attention and emotion are affected. ${ }^{10}$

\section{RELATED WORK}

In a similar vein, studies on meditation have found that calm mental states have the potential to influence time perception. In one study it was found that both inexperienced and experienced meditators exhibited increased post-test performances in a temporal bisection task posttest compared to pretest when they engaged in 10 minutes of meditation prior to engaging in the task. ${ }^{11}$ Additionally, all participants exhibited decreased anxiety, as well as self-reports of slowed perception of time, following meditation. ${ }^{11}$ Demonstrating the effects of increased anxiety was a study that explored the relationship between nicotine withdrawal and duration discrimination ability by having habitual smokers abstain from smoking for 24 hours prior to the study. ${ }^{11}$ Given the wellknown negative psychophysiological effects that accompany nicotine withdrawal (e.g., anxiety, attentional disruption), the authors hypothesized that duration discriminability would be overestimated. ${ }^{12}$ Results of this study indicated that all participants were more likely to overestimate the passage of time when they abstained from smoking and that this effect was stronger in males than females. ${ }^{13}$

\section{TIME PERCEPTION AND EMOTIONAL AROUSAL}

Apart from the indirect evidence, a number of studies directly support the notion that emotional arousal disrupts time perception processes. For example, in one study subjects were administered the State-Trait Anxiety Inventory (STAI) and then asked to engage in a duration prediction task. ${ }^{14}$ Specifically, the participants had to estimate how long it would take to read a five-page manuscript. Results of this study indicated that the highest STAI scores were correlated with greater prediction overestimations. These results were in accord with past research suggesting that anxiety is associated with negative expectation biases, and indicates that individuals with higher levels of anxiety may anticipate greater cognitive processing demands. ${ }^{14}$ Similarly, in another using the same duration prediction task as the previous study, it was found that individuals who are more conscientious are also more likely to overestimate how long it will take them to complete the task. ${ }^{15,16}$ This result suggests a mediating link between anxiety and conscientious; however, the temporal ordering of these variables is not unambiguously dissociable. Given these effects are due to negative emotional biases, these results can be linked back to aforementioned studies in which it was found that negative emo- 
tional stimuli have the potential to attenuate attentional resources. ${ }^{9}$ Taken together, the idea that emotional arousal distorts time perception ability is strongly supported.

\section{DURATION DISCRIMINATION}

Although attentional resources and emotional arousal are interdependent processes mediated by shared brain mechanisms, and those relationships likely impact time perception, different questions remain unanswered: specifically, how accurately are interval durations judged compared to actual clock time durations and how accurately are shorter interval durations compared to longer durations? Providing partial answers to these questions a study examined how accurately interval durations are estimated as a function of interval duration length. ${ }^{17}$ Each participant was asked to estimate each of five time intervals (e.g. 15, 30, 45, 60 and 75 seconds). The results of this study indicated that time estimation was significantly affected by interval duration length and the shorter interval durations were judged more accurately than longer durations. Subsequent studies using similar paradigms indicate that shorter interval durations are judged more accurately than longer durations. ${ }^{18}$ While these studies support the notion that working memory is a key component of time perception, and is possibly the reason for what constrain the ability to estimate time, they do not account for the impact that emotions can have on the ability to estimate time. ${ }^{18}$

\section{PRESENT STUDY}

If attention and emotional arousal are interdependent processes that affect time perception, how those two variables affect one another during the estimation of time over varying interval duration lengths could evince their relationship. The aforementioned studies described that while humans have the ability to track clock time, their perceived time is not necessarily a reflection of actual clock time. The models and theories of time perception discussed primarily focus on the time estimation aspect of time perception. Though humans have the ability to discriminate interval durations accurately, those interval discriminations are susceptible to distortion by an individual's emotional state. In particular, the emotional state of anxiety appears to disrupt an individual's attentional resources. Because emotional states normally persist longer than durations previously studied, studying these effects over prolonged durations will improve further delineate the impact of emotional arousal on the ability to estimate time.

The following study was designed to examine the effect of anxiety on time estimation and to determine if accurate time estimation judgments are affected by interval duration. The interval durations utilized in this study were selected based on their being significantly longer than the durations used in similar studies; the interval durations selected for this study were one, three, and five minutes. The researcher hypothesized that higher levels of state anxiety would affect participants in such a way that they would overestimate the passage of time. In addition, it was also hypothesized that shorter time interval durations would be estimated more accurately than longer durations.

\section{METHOD}

\section{Participants}

Thirty participants (22 female, 8 male) were recruited from psychology classes and the cafeteria at Coastal Carolina University using a non-random convenience sampling technique. Institutional IRB approved this project. Subjects ranged in age from 18 to 27 years $(\mathrm{M}=21.3)$. Participants were compensated by their professors with bonus points added to their lowest exam grade.

\section{Materials}

A standard informed consent form was used which was a part of each materials packet. A demographic survey was also used in order to obtain participant's general information such as their sex, age, and academic major. A materials packet also included the state-anxiety portion of the State-Trait Anxiety Inventory (STAI) ${ }^{19}$ The STAI differentiates between two types of anxiety; state anxiety and trait anxiety, where state anxiety and trait anxiety are both defined as emotional conditions that are characterized by apprehension, tension, and fear about a particular situation. ${ }^{19}$ The primary difference between state and trait anxiety is that trait anxiety is a more continuous form of anxiety, while state anxiety is temporary. Furthermore, those with trait anxiety are persistently expectant of situations that might create anxiety, which further exacerbates this condition. The STAI is a self-report assessment survey designed to measure both state anxiety and trait anxiety. There are two sections of the STAI. One section of the STAI is designed to measure state-anxiety and the other section is designed to measure trait-anxiety. Both sections of the STAI contain 20 response items such as "I feel frightened" or "I feel calm". Participants respond to those response items by circling a response from a four-point Likert scale in which one refers to "NOT AT ALL" and four refers to "VERY MUCH SO". Those Likert responses are designed to describe how the participant presently feels. ${ }^{19}$ A Unisex Timex T53151 stopwatch was used to time response intervals.

\section{Procedure}

Data were collected in a lab in the psychology department at Coastal Carolina University. The lab was a small room that was lit with standard fluorescent lighting and it contained one desk and two chairs which were placed at opposite sides of the desk. Upon arrival at the laboratory, each participant was welcomed and asked to be seated. Once seated, the participant was handed a materi- 
als packet that contained an informed consent form, demographic survey, and a copy of the state-anxiety portion of the STAI. Upon completion of their materials, those were collected. Each participant was randomly assigned to one of three interval groups of either one, three, or five minutes. The participant was instructed that he/she would estimate an interval of time and that the use of cellphones, watches, or any other electronic device was not permitted. In addition, the participant was also told he/she could not count aloud or use rhythmic tapping to estimate the time interval.

The participant was instructed that the activity would begin when the researcher said aloud "NOW" and that when the participant estimated the time had elapsed the participant was to say "OK" or "STOP" aloud. The researcher sat across the table from the participant and utilized a stopwatch to record the participant's estimate of the time interval. The stopwatch was activated when the participant said "NOW" and recorded time in seconds until the participant said "STOP". Upon completion of the activity the participant was thanked for his/her participation and escorted from the lab. These procedures were repeated for all 30 participants in this study.

\section{RESULTS}

This study investigated the dependent variables of participant-estimated time intervals and state-anxiety scores. The highest score on the state-anxiety portion of the STAI is 80 and the lowest score is 20. In this study, state-anxiety scores ranged between 20 and 60. The average state-anxiety scores for males was 35.00 and the standard deviation was 12.78 and average state-anxiety score for females was 38.09 and the standard deviation was 10.38. A second dependent variable in this study was an absolute error (AE), and these values represented the difference between participant-estimated time intervals and experimenter-assigned time intervals. The independent measure in this study was the experimenter-assigned time interval. For participants in the one-minute experimenter-assigned time interval group, the average time estimate was 64.00 seconds and the standard deviation was 24.23 seconds. Participants in the three-minute experiment assigned interval group the average time estimate was 170.60 seconds and the standard deviation was 46.09 seconds. For participants in the five-minute experimenter-assigned interval group, these values were 296.00 seconds and 76.50 seconds respectively. (See Table 1 for experimenter-assigned time intervals compared to the average estimate of assigned time intervals.)

\begin{tabular}{|c|c|}
\hline Experimenter-Assigned Time Intervals & $\begin{array}{c}\text { Average Estimate of Assigned Time Inter- } \\
\text { vals }\end{array}$ \\
\hline one minute (60 seconds) & 64.00 seconds \\
\hline three minutes (180 seconds) & 170.60 seconds \\
\hline five minutes (300 seconds) & 296.00 seconds \\
\hline
\end{tabular}

Table 1. Experimenter assigned time intervals compared to the average estimates of assigned time intervals.

It was hypothesized that higher state-anxiety scores would correspond with participant overestimations of time interval durations. The average state-anxiety score for those who overestimated the time intervals was 37.77 and the standard deviation was 8.85 and for those who underestimated the time intervals the average state-anxiety score was 36.12 and the standard deviation was 12.34. In order to determine if state-anxiety scores differed as a function of over or underestimations of time, an independent-samples one-tailed t-test was calculated to compare scores from 13 participants who overestimated and 17 participants who underestimated their assigned time intervals (refer to Table 2 for group sizes, mean state-anxiety scores, and standard deviations as a function of over or underestimation groups). The results of this test indicated no statistical difference in state-anxiety scores between participants who overestimated and those who underestimated assigned time intervals, $t(28)=-.41, \mathrm{P}>.05$.

\begin{tabular}{|l|l|l|l|}
\hline STP & $\mathbf{n}$ & MSA & sd \\
\hline Overestimated & 13 & 37.77 & 8.85 \\
\hline Underestimated & 17 & 36.12 & 12.34 \\
\hline
\end{tabular}

Table 2. Group sizes, mean State-Anxiety Scores, and standard deviations as a function of over or underestimation groups. 
Following the elimination of three extreme outliers, state-anxiety and general time-estimation error were tested by conducting a correlation analysis. The results of that correlation test revealed a significant negative correlation between state-anxiety score and general time-estimation error, $r=-.33, \mathrm{P}<.05$, one-tailed. An even further examination of the data via a curvilinear regression analysis revealed a substantial curvilinear trend with a significant quadratic term, $t(24)=2.26, \mathrm{P}<.05$ (refer to Figure 1 for graph delineating the curvilinear regression analysis).

To test the hypothesis that shorter interval durations would be estimated more accurately than longer durations a linear trend analysis was calculated between time interval duration and the absolute value of the error of time estimations. Results of this test indicated statistical significance for the effect of interval duration length and accuracy of participant-estimated time, $F(1,27)=$ 6.12, $\mathrm{P}<.05$ (refer to Figure 2 for graph delineating the linear trend analysis).

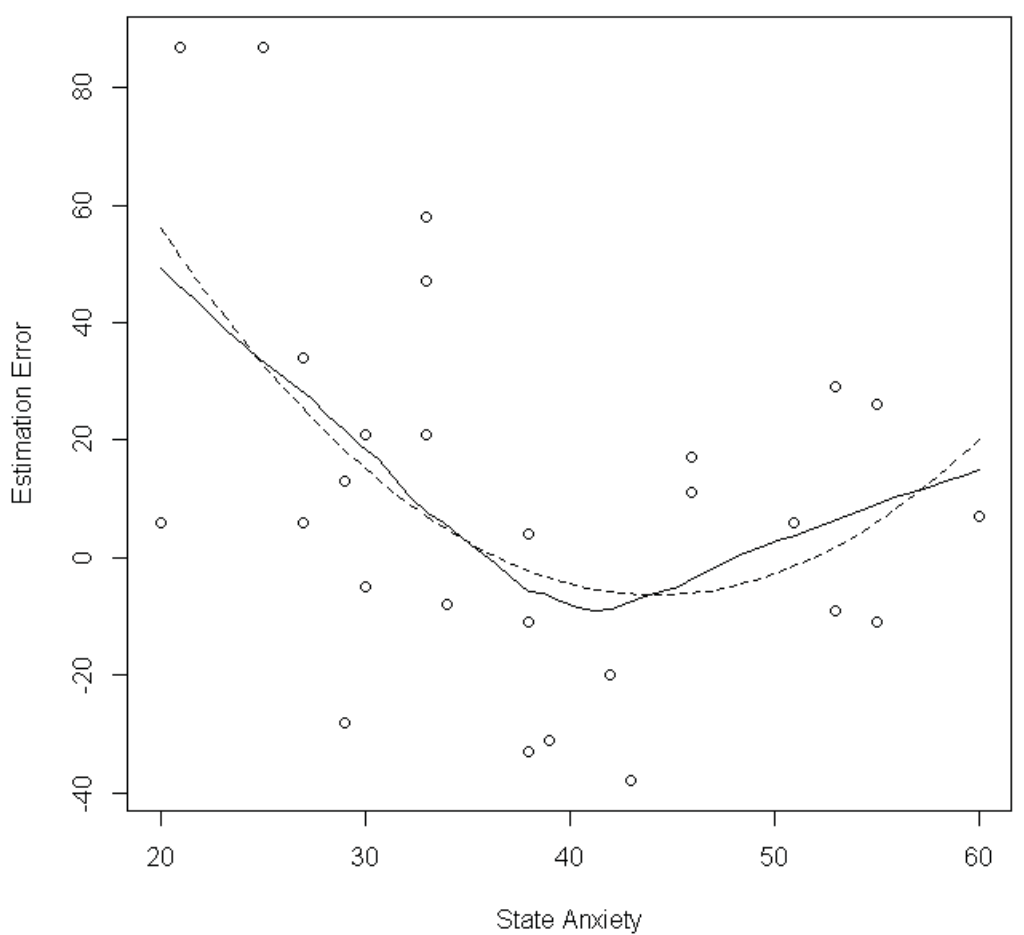

Figure 2. Curvilinear regression analysis delineating a substantial curvilinear trend between state-anxiety score and general time-estimation error.

\section{DISCUSSION}

It was predicted that higher state-anxiety scores would correspond with participants overestimating their assigned interval durations. The results of a t-test did not indicate that higher state-anxiety scores corresponded with an overestimation of interval durations. Though the hypothesis was not supported, the results of a multiple regression analysis did indicate that there was an effect for state-anxiety scores on participant-estimated time error. The curvilinear regression analysis was calculated to examine differences between participants with highest state-anxiety scores and participants with lowest state anxiety scores as a function of participant-estimated time error. When the data were analyzed to find if there were any effects for state-anxiety score and general time-estimation error, a significant negative correlation was found in the first analysis between those two variables. The discovered relationship appeared to be due to a very strong negative correlation in participants with anxiety scores less than 45 . The results of the quadratic curvilinear test agree with the results of the correlation test but go further by suggesting that participants whose state-anxiety scores were between 30 and 50 had the least amount of error when estimating their interval durations, compared to participants with both the lowest and the highest state-anxiety scores. While both the lowest and the highest state-anxiety scores were associated with more error, it should be noted that the participants with lowest state-anxiety scores had more timeestimation error than those with the highest state-anxiety scores.

The effects of anxiety on time perception reported here are bidirectionally consistent with previous work and strongly suggest that valenced emotional states, whether positive or negative, interfere with available attentional resources and distort time perception. However, the findings of the present study indicate that both low and high emotional arousal distorts the availability of attentional resources. These findings are consistent with the accumulator pacemaker models of time perception because emotional arousal disrupts attentional resources which complicate time estimation ability. While there was a curvilinear relationship between stateanxiety score and general time-estimation error, it is important to interpret this information with caution because it is uncertain 
why the highest and lowest state-anxiety scores had more time estimation error than mid-level state-anxiety scores. The data seems to indicate that in order to estimate time accurately a person has to be calm, but not so calm that they lose focus on the interval they are estimating, and perhaps a certain degree of anxiousness could be the result of prolonged focus, though this is more speculation than an empirically based assertion. The results of a linear trend analysis revealed a statistically significant positive linear relationship between interval duration and participant estimation error. Longer duration intervals were related to more participant-estimated error. The findings of this study are consistent with the findings of previous studies, even though other studies typically utilized shorter interval durations (e.g. one, three, seven, and 20 seconds). ${ }^{17,} 18$ Researchers from past studies have reported that with longer interval durations there was more participant-estimated time error. ${ }^{17}$, 18 Perhaps longer interval durations are harder for the brain to process than shorter durations because of attentional resource limitations, though the exact cause of this phenomenon is still unknown.

Although there were statistically significant results attained in this study there were some limitations. A limitation of this study was the relatively small sample size. There were only 30 participants. For comparison, similar studies done in the past have involved as many as 100 participants. ${ }^{16}$ In addition to a small sample size, the researcher only recruited college students to participate, which significantly skewed the age demographic to represent primarily young adults. It should also be noted a few technical challenges could have compromised the validity of this study. During participant testing, the researcher was present in order to record partic ipants' time estimates. After a few participants had completed their time estimations, they informed the researcher that sitting silently in the room while the researcher sat across from them was awkward. Since awkwardness is an unpleasant sensation, those negative emotions may have disrupted the participants' attentional resources, thus confounding their ability to estimate time. In order to avoid this issue in the future, it would be best if the researcher were absent from the room while the participants estimated their assigned time intervals. Perhaps a reaction time button could be pressed by participants and times could be recorded automatically in the absence of the researcher. In this manner, the researcher-participant proximity awkwardness would be eliminated. Because the researcher had to manually measure each participant's response using a stopwatch, the researcher was neither blind to who was participating in the study nor blind to which interval group a particular participant belonged in. Thus, the method by which the experiment was conducted could have influenced the findings, as the presence of the researcher in the room could have caused participants to become more relaxed or more anxious.

\section{FUTURE STUDIES}

Future modifications to the study could include the addition of distractions, such as having one group of participants engage in something they consider to be a fun or enjoyable activity (ex: playing a video game) and having the other group engage in something they consider a boring activity (ex: reading a chemistry textbook). These distractions could test the popular phrase "time flies by when you're having fun." However, it would be necessary to ask participants to disclose whether they actually found the activity enjoyable or boring as these can differ between participants. While only the emotional state of anxiety was examined in this study, psychological disorders that interfere with attentional resources and emotional arousal may also affect human time perception, such as attention-deficit hyperactivity disorder (ADHD) and depression. In ADHD, attentional resources are diminished, which may affect time perception. In fact, studies on ADHD and time perception have indicated statistically significant duration discrimination deficits in children with ADHD, in that children with ADHD are more likely to make discrimination errors. ${ }^{20}$

\section{CONCLUSION}

The present study demonstrated significant results for the effects of interval duration and state-anxiety on time perception. In a time-based society where human behavior is often governed by deadlines and time parameters, modern human existence is profoundly affected and measured by the clock. Even with the invention of watches and cell phones to inform humans minute by minute of the time, it is still a challenge to conform to the unchanging rigidity of clock time. Continued study of time perception will not only broaden scientific understanding of how and why human time perception differs from real time, but may also garner knowledge that may help humans make better use of their time and effectively improve their quality of life.

\section{ACKNOWLEDGEMENTS}

The author of this paper thanks Dr. Joan Piroch and Dr. William Kind for their mentorship in guiding the development of this paper. 


\section{REFERENCES}

1. Tanaka, H., Homma, K., \& Imamizu, H. (2012). Illusory reversal of causality between touch and vision has no effect on prism adaptation rate. Frontiers in Psychology, 3, 545.

2. Grondin, S., \& Rousseau, R. (1991). Judging the relative duration of multimodal short empty time intervals. Perception and Psychophysics, 49(3), 245-256.

3. Gibbon, J. (1977). Scalar expectancy theory and Weber's law in animal timing. Psychological review, 84(3), 279.

4. Fortin, C., \& Couture, E. (2002). Short-term memory and time estimation: Beyond the 2-second "critical" value. Canadian Journal of Experimental Psychology, 56(2), 120-127.

5. Wencil, E. B., Coslett, H. B., Aguirre, G. K., \& Chatterjee, A. (2010). Carving the clock at its component joints: neural bases for interval timing. Journal of neurophysiology, 104(1), 160-168.

6. Kojima, Y. \& Matsuda, F. (2000). Effects of attention and external stimuli on duration estimation under a prospective paradigm. Japanese Psychological Research, 42(3), 144-154.

7. Buhusi, C. V., \& Meck, W. H. (2005). What makes us tick? functional and neural mechanisms of interval timing. Nature Reviews Neuroscience, 6(10), 755-765.

8. Bush, G., Luu, P., \& Posner, M. I. (2000). Cognitive and emotional influences in anterior cingulate cortex Elsevier Ltd.

9. Carretie, L., Hinojosa, J.A., Martin-Loeches, M., Mercado, F., \& Tapia, M. (2004). Automatic attention to emotional stimuli: Neural correlates. Human Brain-Mapping, 22(4), 290-299.

10. Manuello, J., Vercelli, U., Nani, A., Costa, T., \& Cauda, F. (2016). Mindfulness meditation and consciousness: An integrative neuroscientific perspective. Consciousness and Cognition, 40, 67-78.

11. Droit-Volet, S., Fanget, M., \& Dambrun, M. (2015). Mindfulness meditation and relaxation training increases time sensitivity. Consciousness and Cognition, 31, 86-97.

12. Carrasco, M.C., \& Redolat, R., \& Simon, V.M. (1998). Effects of cigarette smoking on time estimation. Human Psychopharmacology, 13, 565-573.

13. Ashare, R. L., \& Kable, J. W. (2015). Sex Differences in Time Perception During Smoking Abstinence. Nicotine \& Tobacco Research, 17(4), 449-454.

14. Kelly, W. E. (2002). Anxiety and the prediction of task duration: A preliminary analysis. The Journal of Psychology, 136(1), 53-58.

15. Kelly, W. E., Johnson, J. L., \& Miller, M. J. (2003). Conscientiousness and the prediction of task duration. North American Journal of Psychology, 5(3), 443.

16. Kelly, W. E. (2004). College Students' Accuracy and Perceptions of Accuracy in Predicting the Duration of an Academic-Related Task. Individual Differences Research, 2(3), 225-230.

17. Koivula, N. (1995). Estimation of time: Effects of locus of control, mental arithmetic, and length of target interval. Personality, Individual Differences, and Intelligence, 20(1), 25-32.

18. Hancock, P.A., \& Raush, R. (2010). The effects of sex, age, and interval duration on the perception of time. Acta Psychologica, 133, 170-179.

19. Spielberger, C. D., Gorsuch, R.L., and Lushene. R.E. (1970). Manual for the State-Trait Anxiety Inventory. Palo Alto, CA: Consulting Psychologists Press.

20. Plummer, C., \& Humphrey, N. (2009). Time perception in children with ADHD: The effects of task modality and duration. Child Neuropsychology, 15, 147-162.

\section{ABOUT THE STUDENT AUTHOR}

The author completed this research as an undergraduate studying psychology at Coastal Carolina University during May of 2011. Currently, he's a graduate student at in the biological psychology program at Virginia Tech studying visual neuroscience and brain imaging.

\section{PRESS SUMMARY}

Time perception underlies our ability to understand the temporal order of causal events. While time perception is a fundamental of learning and human behavior, evidence suggests our perceptions of time are subject disturbances incurred by our emotional states. Thus, our emotional states, which can alter by a multitude of internal and external phenomena, to some degree dictate our perception of time and ultimately our ability to learn and interact with the world. 